\section{Pre-emptive screening for peanut allergy before peanut ingestion in infants is not standard of care}

We read with interest the article by Kalaichandran and colleagues ${ }^{1}$ about early introduction of allergenic solids. We appreciate this focus, as it has been shown that early introduction of both peanut and cooked egg significantly reduce the risk of peanut and egg allergy, respectively. ${ }^{2}$

Our main concern is with point no. 4 , which recommends that infants with risk factors see a specialist for skin-prick or specific immunoglobulin $\mathrm{E}$ testing before peanut introduction. We appreciate that in the Learning Early About Peanut (LEAP) study, pre-emptive screening before peanut ingestion was routine for all high-risk infants who participated. ${ }^{3}$ Although this approach was a requirement of the research protocol, it is not yet established as a standard of care. The recent Canadian Paediatric Society practice point on food allergy prevention ${ }^{4}$ makes no mention of pre-emptive screening before peanut introduction. This recommendation is also not present in guidance endorsed by the Australian ${ }^{5}$ or British ${ }^{6}$ national allergy societies.

The most important barriers to widespread adoption of pre-emptive allergen testing are the lack of feasibility outside of research, false-positive results and the heavy burden related to overtesting and overdiagnosis. An Australian study estimated that pre-emptive screening of all infants potentially considered "high risk" for peanut allergy would result in screening $16 \%$ of the infant population while still missing $23 \%$ of peanut allergy cases. ${ }^{7}$ A Canadian survey that we performed noted that most allergists, pediatricians and family physicians do not suggest preemptive testing before peanut introduction and that most allergists are not comfortable following up on moderately positive allergy testing. ${ }^{8,9}$ Concerns have also been raised that pre-emptive testing may result in a "screening creep" and inadvertently result in delaying peanut introduction. ${ }^{10}$

Substantial systemic barriers exist for carrying out oral food challenges, as we recently described in the first Canadian study of implementing oral food challenges. ${ }^{11}$ Paradoxically, infants with no history of peanut ingestion but positive skin-prick tests (i.e., high potential for false-positives) who are never offered oral food challenges will be placed at increased risk of developing peanut allergy resulting from prolonged peanut avoidance. Other infants with positive skin-prick tests placed on long waitlists for infant oral food challenges could completely bypass the window of opportunity for primary prevention, again increasing risk.

Although Kalaichandran and colleagues suggested specific amounts of peanut ingestion, ${ }^{1}$ to date there are inconclusive data regarding the exact amount and periodicity of peanut protein ingestion required to prevent peanut allergy. The LEAP study used a protocol of $6 \mathrm{~g}$ of peanut protein per week distributed over 3 or more meals, ${ }^{2}$ but this has never been replicated, and to date, no study has tested whether a smaller amount would give the same protection. Current Canadian guidance recommends feeding peanut a few times a week once introduced, if well tolerated, but does not specify exact amount or frequency, as that remains unknown. ${ }^{4}$

We believe the recent Canadian Paediatric Society practice point on food introduction strikes a more appropriate balance of what is known and what is feasible in a Canadian context, and we discourage any messages that promote blanket pre-emptive screening for any risk category. The key simple message for health care providers and parents is to introduce peanut and other common allergens at around 6 months but not before 4 months of age, at home (especially if a child is atopic or has a family history of allergies), and to keep these foods in the diet on a regular basis if well tolerated. ${ }^{4}$

\section{Elissa M. Abrams MD}

Pediatric allergist, Department of Pediatrics, Section of Allergy and Clinical Immunology, University of Manitoba, Winnipeg, Man.

\section{Alexander G. Singer MB BCh BAO}

Family physician, Department of Family

Medicine, University of Manitoba,

Winnipeg, Man.

\section{Edmond S. Chan MD}

Pediatric allergist, Department of Pediatrics, Division of Allergy and Immunology, University of British Columbia, Vancouver, BC

Cite as: CMAJ 2019 October 21;191: E1169-70. doi: 10.1503/cmaj.73447

\section{References}

1. Kalaichandran A, Marrs T, du Toit G. Early introduction of infant-safe peanut protein to reduce the risk of peanut allergy. CMAJ 2019;191:816.

2. Ierodiakonou D, Garcia-Larsen V, Logan A, et al. Timing of allergenic food introduction to the infant diet and risk of allergic or autoimmune disease: a systematic review and meta-analysis. JAMA 2016;316:1181-92.

3. Du Toit G, Roberts G, Sayre PH, et al. Randomized trial of peanut consumption in infants at risk for peanut allergy. N Engl J Med 2015;372:803-13.

4. Abrams EM, Hildebrand KJ, Blair B, et al. Timing of introduction of allergenic solids for infants at high risk. Paediatr Child Health 2019;24:56-7.

5. Netting MJ, Campbell DE, Koplin JJ, et al. An Australian consensus on infant feeding guidelines to prevent food allergy: outcomes from the Australian infant feeding summit. J Allergy Clin Immunol Pract 2017;5:1617-24.

6. Preventing food allergy in higher risk infants: guidance for healthcare professionals [information sheet]. London (UK): British Society for Allergy \& Clinical Immunology; 2018. Available: www.bsaci.org/pdf/Early-feeding-guidance-for -HCPs.pdf (accessed 2019 Mar. 24)

7. Koplin JJ, Peters RL, Dharmage SC, et al. Under standing the feasibility and implications of implementing early peanut introduction for prevention of peanut allergy. J Allergy Clin Immunol 2016;138:1131-1141.e2.

8. Abrams EM, Singer AG, Soller L, et al. Knowledge gaps and barriers to early peanut introduction among allergists, pediatricians, and family physicians. J Allergy Clin Immunol Pract 2019;7:681-4.

9. Abrams EM, Soller L, Singer AG, et al. Comparison of practice patterns among Canadian allergists before and after NIAID guideline recommendations. $J$ Allergy Clin Immunol Pract 2019 May 14 [Epub ahead of print]. doi:10.1016/j.jaip.2019.04.044 
10. Turner PJ, Campbell DE. Implementing primary prevention for peanut allergy at a population level. JAMA 2017;317:1111-2.

11. Hsu E, Soller L, Abrams EM, et al. Oral food challenge implementation: the first mixed-methods study exploring barriers and solutions. J Allergy Clin Immunol Pract 2019 July 10 [Epub ahead of print]. doi :10.1016/j.jaip.2019.06.034

Competing interests: Elissa Abrams reports a role as medical advisor for Food Allergy Canada, and was first author on the Canadian Paediatric Society Practice Point on Food Allergy Prevention, outside the submitted work. Alexander Singer reports receiving funding from IBM and Calian, and a research grant administered by the Canadian Institute for Military and Veterans Health Research, outside the submitted work. Edmond Chan reports that he is a member of the health care advisory board for Food Allergy Canada, and was an expert panel and coordinating committee member of the National Institute of Allergy and Infectious Diseases-sponsored Guidelines for Peanut Allergy Prevention, outside the submitted work. He also reports receiving a grant from DBV Technologies, and personal fees from Pfizer, Kaleo, Pediapharm and Leo Pharma. No other competing interests were declared. 